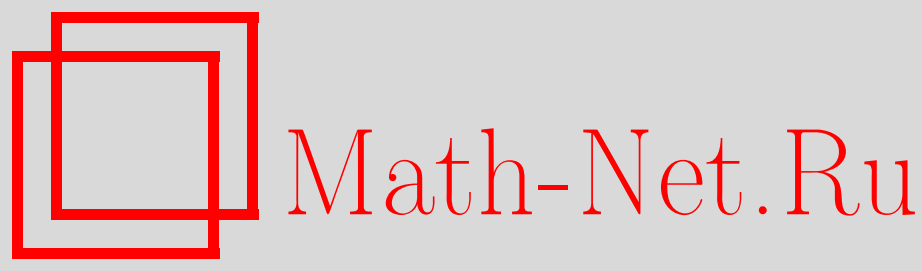

А. С. Трушечкин, Необратимость и роль измерительного прибора в функциональной формулировке классической механики, ТМФ, 2010, том 164, номер 3, 435-440

DOI: https://doi.org/10.4213/tmf6554

Использование Общероссийского математического портала Math-Net.Ru подразумевает, что вы прочитали и согласны с пользовательским соглашением http://www.mathnet.ru/rus/agreement

Параметры загрузки:

IP: 54.80 .97 .219

26 апреля 2023 г., 13:50:21

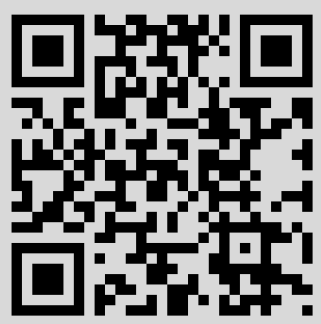




\title{
НЕОБРАТИМОСТЬ И РОЛЬ ИЗМЕРИТЕЛЬНОГО ПРИБОРА В ФУНКЦИОНАЛЬНОЙ ФОРМУЛИРОВКЕ КЛАССИЧЕСКОЙ МЕХАНИКИ
}

\begin{abstract}
Анализируется роль прибора в недавно предложенной функциональной формулировке классической механики, основным уравнением которой является уравнение Лиувилля. Его решения обладают свойством делокализации (расплывания), что интерпретируется как необратимость на микроуровне. Показывается, что, отслеживая динамику частицы с помощью приборов, можно наблюдать приближенно обратимую и возвращаемую динамику для частицы, однако многократные измерения неизбежно приводят к выделению тепла и возрастанию энтропии в приборе. Таким образом, необратимое поведение переносится с изучаемой системы на измерительный прибор, который тоже является физической системой.
\end{abstract}

Ключевые слова: классическая механика, проблема необратимости, уравнение Лиувилля, теория измерений.

1. В статье Воловича [1] предлагается новая формулировка классической механики, названная функциональной. Исходное понятие механики в этой формулировке не материальная точка, а функция плотности распределения в фазовом пространстве. Соответственно фундаментальным динамическим уравнением становится не уравнение Ньютона (или эквивалентные ему уравнения Гамильтона), а уравнение Лиувилля, даже если речь идет не об ансамбле, а об одной частице. Уравнение Ньютона (Гамильтона) тогда становится приближенным уравнением для средних координат и импульсов. В работе [1] вычислены поправки к решениям уравнения Ньютона для некоторых частных случаев.

Целью перехода к новой формулировке классической механики является согласование обратимой микроскопической динамики с необратимой макроскопической. Эта проблема известна как проблема необратимости и является в настоящее время

* Математический институт им. В. А. Стеклова РАН, Москва, Россия.

E-mail: trushechkin@mi.ras.ru

${ }^{\dagger}$ Национальный исследовательский ядерный университет "МИФИ”, Москва, Россия. 
одной из важнейших фундаментальных проблем математической физики, требующих своего решения. Как известно, динамика материальной точки обратима и возвращаема. Но динамика функции плотности, подчиняющаяся уравнению Лиувилля, обладает так называемым свойством делокализации (расплывания): если частица движется в ограниченном объеме (например, ящике), то на больших временах достигается равномерное распределение пространственной плотности (см. теоремы Козлова о диффузии в бесстолкновительной сплошной среде [2], [3]). Это свойство соответствует необратимому поведению. Поэтому, если мы описываем с помощью функции плотности не только системы, состоящие из большого числа частиц, но даже и одну частицу, то противоречия между микро- и макроскопической динамиками нет (см. также [4]): обе динамики необратимы.

Аргумент в пользу функциональной формулировки состоит в том, что произвольные вещественные числа, т.е. бесконечные дроби (а с ними и отдельные траектории) не наблюдаемы. Поэтому в самом деле рассмотрение пучка траекторий или динамики функции плотности распределения более оправданно, чем рассмотрение отдельной траектории точки. Отдельная траектория является своего рода “скрытой переменной" и не имеет непосредственного физического смысла.

2. В работе [5] приводится еще один аргумент в пользу этой позиции: поскольку непосредственно наблюдаемые величины - это показания измерительных приборов, а любой прибор имеет погрешность, то в действительности мы никогда не знаем состояние системы как точку в фазовом пространстве. Мы знаем состояние системы только как некоторое распределение вероятностей. В той же работе предлагается метод построения функции плотности распределения на основе непосредственно наблюдаемых величин - показаний прибора - с помощью методов математической статистики. А именно если $X^{(1)}, \ldots, X^{(n)}$ - результаты измерений некоторой наблюдаемой $\mathbb{X}\left(X^{(1)}, \ldots, X^{(n)}\right.$ - независимые и одинаково распределенные случайные величины), то этой наблюдаемой ставится в соответствие функция плотности

$$
\rho(x)=\frac{1}{\sqrt{2 \pi S^{2}}} e^{-(x-\bar{X})^{2} /\left(2 S^{2}\right)},
$$

где

$$
\bar{X}=\frac{1}{n} \sum_{i=1}^{n} X^{(i)}
$$

- статистическая оценка среднего значения показаний прибора,

$$
S^{2}=\frac{S_{\text {сл }}^{2}}{n}+\sigma_{\text {сист }}^{2}
$$

- оценка дисперсии, связанной с полной погрешностью измерений,

$$
S_{\text {сл }}^{2}=\frac{1}{n-1} \sum_{i=1}^{n}\left(X^{(i)}-\bar{X}\right)^{2}
$$

- оценка дисперсии, связанной со случайной погрешностью, $\sigma_{\text {сист }}^{2}$ - дисперсия, связанная с систематической погрешностью (предполагается известной, так как может быть оценена с помощью данных паспорта прибора). 
3. Настоящая работа посвящена рассмотрению роли измерительного прибора в функциональной формулировке динамики. Пусть имеется частица на прямой. В начальный момент времени $t=0$ мы провели серию измерений координаты и импульса и построили функцию плотности вида

$$
\rho(q, p)=\frac{1}{2 \pi s_{q} s_{p}} \exp \left[-\frac{(q-\bar{q})^{2}}{2 s_{q}^{2}}-\frac{(p-\bar{p})^{2}}{2 s_{p}^{2}}\right] .
$$

Это двумерный аналог формулы (1). Здесь мы перешли от случайных величин оценок средних $\bar{Q}, \bar{P}$ и дисперсий $S_{q}^{2}, S_{p}^{2}$ к конкретным реализациям этих случайных величин (т.е. конкретным значениям, полученным в результате измерений и вычислений) $\bar{q}, \bar{p}, s_{q}^{2}$ и $s_{p}^{2}$.

С течением времени распределение показаний приборов меняется. А именно если в начальный момент времени совместное распределение координаты и импульса частицы приближается функцией $\rho$, то в произвольный момент $t$ оно будет приближаться функцией $\rho_{t}(q, p)$, которая является решением задачи Коши для уравнения Лиувилля с начальным условием $\rho$ :

$$
\frac{\partial \rho_{t}}{\partial t}=-\frac{p}{m} \frac{\partial \rho_{t}}{\partial q}+\frac{\partial V}{\partial q} \frac{\partial \rho_{t}}{\partial p}, \quad \rho_{0}=\rho
$$

здесь $\rho_{t}=\rho_{t}(q, p), V=V(q)$ - потенциальная энергия, $m>0$ - масса.

Для многих потенциалов $V$ (в частности, в случае свободного движения $V=0$ ) с течением времени наблюдается расплывание плотности распределения ("делокализация") [1]. Поэтому даже если в начальный момент времени мы знали положение частицы с очень хорошей точностью, со временем погрешность в определении положения частицы нарастает, и на больших временах сказать, где находится частица, мы практически не можем.

В этих рассуждениях важно, что на рассматриваемом промежутке времени мы больше не выполняли никаких измерений. Таким образом, уравнение Лиувилля описывает динамику функции плотности вероятности при отсутствии повторных измерений. Но как только в момент $t$ мы провели очередную серию измерений координаты и импульса частицы, в результате изменения нашего знания о состоянии функция плотности снова "схлопывается" в функцию вида (2) (конечно, вообще говоря, с другими значениями $\bar{q}, \bar{p}, s_{q}^{2}$ и $s_{p}^{2}$ ). В какой-то степени это классический аналог квантового эффекта Зенона, когда постоянное наблюдение за частицей удерживает ее от неограниченного расплывания. Здесь мы пренебрегаем воздействием прибора на изучаемую систему, которое в классической механике может быть сделано сколь угодно малым, и рассматриваем изменение состояния при измерении только как следствие изменения нашего знания о нем.

Отсюда можно заметить, что при достаточно частых повторных измерениях можно наблюдать приближенно обратимую и возвращаемую динамику: вследствие частых измерений состояние системы будет постоянно схлопываться в состояния вида (2), и неограниченной делокализации не будет. Фактически динамика функции плотности приближенно сведется к динамике средних, т.е. к уравнениям Ньютона 
(Гамильтона), которые обладают свойствами обратимости и возвращаемости. Формализуем рассуждение, обосновывающее возможность наблюдать возвращаемость динамики в функциональной формулировке.

ПреДЛОЖЕНИЕ 1. Пусть $\left(\Omega, \mathcal{F}, \mu, \varphi_{t}\right)$ - динамическая система, где $\Omega$ - некоторое множество, $\mathcal{F}$ - сигма-алгебра измеримых подмножеств $\Omega, \mu$ - мера на $\Omega$, причем $\mu(\Omega)<\infty, \varphi_{t}, t \in \mathbb{R},-$ группа автоморфизмов $\Omega$. Пусть далее на измеримом пространстве $(\Omega, \mathcal{F})$ задана также некоторая вероятностная мера $P$, абсолютно непрерывная относительно меры $\mu$. И пусть $G \in \mathcal{F}-$ измеримое подмножество $\Omega$. Тогда для любого вещественного $Т$ выполнено

$$
P\left(\bigcup_{t>T} \varphi^{t}(G)\right) \geqslant P(G) .
$$

Предложение 1 фактически является следствием теоремы Пуанкаре о возвращении. Поясним его смысл. Пусть дана динамическая система, например, одна или несколько частиц в закрытом ящике, $\varphi^{t}$ - гамильтонов поток, $\Omega$ - поверхность энергетического уровня, $\mu$ - инвариантная мера, и выполнено условие теоремы Пуанкаре $\mu(\Omega)<\infty$ (оно заведомо выполнено, например, если частицы не взаимодействуют друг с другом). Выберем множество $G$ так, чтобы оно соответствовало случаю, когда все частицы собраны в одной из половин ящика (фиксированной). Вероятностная мера $P$ на $\Omega$ связана с нашей оценкой начального состояния. Мера $P$, будучи абсолютно непрерывной относительно $\mu$, имеет интегрируемую плотность $\rho$. На практике, как говорилось ранее, функция плотности $\rho$ строится на основе серии измерений в момент времени $t=0$, т.е. в начальный момент времени состояние известно только в виде некоторой интегрируемой функции плотности вероятности. Допустим, известно, что достоверно или с очень большой вероятностью все частицы собраны в фиксированной половине ящика (т.е. $P(G)=1$ или $P(G)=1-\varepsilon$, где $\varepsilon-$ некоторое малое число). Поскольку нам не известно состояние как точка в фазовом пространстве, мы не можем точно определить и время возвращения системы в состояние, когда все частицы снова будут собраны в той же половине ящика (можно назвать его "макросостоянием"). Но когда-то такой момент времени наступит, поэтому, если мы будем непрерывно (или в достаточно частые дискретные моменты времени) наблюдать за системой, то с большой вероятностью в некоторый момент мы заметим это возвращение в начальное макросостояние.

Таким образом, может показаться, что рассмотрение функции плотности вероятности не меняет обратимый и возвращаемый характер динамики, пусть и в приближенном смысле, если над системой производить достаточно частые измерения.

4. Однако при внимательном анализе следует заметить, что многократные измерения не являются "безобидными" с точки зрения проблемы необратимости. В работах [6], [7] при анализе известного мысленного эксперимента "демон Максвелла" указывается на то, что измерение можно провести обратимым образом, но термодинамически необратимой является операция приведения прибора после измерения в исходное состояние. Ее можно также назвать операцией стирания предыдущего результата измерения. Если прибор обладает памятью, то все равно к стиранию старых результатов измерений рано или поздно необходимо прибегать, поскольку 
память любого прибора конечна. В работах [7], [8] показывается, что в результате стирания одного бита информации в некотором устройстве в нем выделяется теплота в количестве не менее $k T \ln 2$, где $k$ - постоянная Больцмана, $T$ - температура устройства. При этом энтропия устройства увеличивается на величину не менее $k \ln 2$.

Определим количество бит, которое необходимо для запоминания результата измерения и которое, следовательно, необходимо стереть перед следующим измерением для того, чтобы привести прибор в исходное состояние.

Пусть $\varepsilon-$ точность, с которой прибор проводит измерение, т.е. $\sigma_{\text {сист }}=\varepsilon$ (для простоты предположим, что случайной погрешности нет). Тогда для запоминания результата необходимо $-\log _{2} \varepsilon$ двоичных разрядов (бит). И в самом деле, количество разрядов в цифровых приборах и цена наименьшего деления в аналоговых приборах, как правило, согласованы с погрешностью самого прибора (поэтому, если класс точности прибора неизвестен, то за погрешность принимают единицу наименьшего разряда для цифровых приборов и половину цены наименьшего деления для аналоговых) [9]. Таким образом, при выполнении одного измерения некоторой одномерной физической величины с точностью $\varepsilon$ в приборе возрастает энтропия минимум на

$$
\Delta S=-k \ln 2 \log _{2} \varepsilon=-k \ln \varepsilon
$$

Таким образом, доказано следующее предложение.

ПРЕДЛОЖЕНИЕ 2. При проведении каждого измерения произволъной одномерной наблюдаемой с величиной систематической ошибки в в измерительном приборе въделяется теплота в количестве не менее $Q=-k T \ln \varepsilon$ и возрастает энтропия на величину не менее $\Delta S=-k \ln \varepsilon$.

Чем более динамическая система хаотична, тем бо́льшую неопределенность имеет время возвращения, тем дольше мы должны проводить частые измерения микроили макроскопических величин, чтобы обнаружить возвращаемость, тем быстрее возрастает энтропия измерительного прибора. Таким образом, теоретически мы можем наблюдать приближенно обратимую и возвращаемую динамику изучаемой физической системы, но только ценой многократных измерений и связанных с ними выделением тепла и возрастанием энтропии в приборе, т.е. необратимое поведение переносится с изучаемой системы на измерительный прибор, который тоже является физической системой.

Благодарности. Работа выполнена при частичной финансовой поддержке РФФИ (гранты № 08-01-00727-а и № 09-01-12161-офи_м) и Программы поддержки ведущих научных школ (грант НШ-7675.2010.1). Автор благодарен И. В. Воловичу за полезные замечания.

\section{Список литературы}

[1] И.В. Волович, Вестн. СамГУ. Естественнонаучная сер., 8/1:67 (2008), 35-55; I. V. Volovich, Time irreversibility problem and functional formulation of classical mechanics, 2009, arXiv: 0907.2445. 
[2] В. В. Козлов, Тепловое равновесие по Гиббсу и Пуанкаре, Современная математика, РХД, М., Ижевск, 2002.

[3] В. В. Козлов, Ансамбли Гиббса и неравновесная статистическая механика, РХД, М., Ижевск, 2008.

[4] В. В. Козлов, Д. В. Трещёв, ТМФ, 151:1 (2007), 120-137.

[5] A.S. Trushechkin, I. V. Volovich, P-Adic Numbers Ultrametric Anal. Appl., 1:4 (2009), 361-367, arXiv: 0910.1502.

[6] L. Szilard, Z. f. Physik, 53:11-12 (1929), 840-856.

[7] C. H. Bennett, Internat. J. Theoret. Phys., 21:12 (1982), 905-940.

[8] Р. Ландауэр, "Необратимость и выделение тепла в процессе вычислений", Квантовый компъютер и квантовые вычисления. т. 1, 2, ред. В.А. Садовничий, Удмуртский университет, Ижевск, 1999, 9-32.

[9] Б. Д. Агапьев, В. Н. Белов, Ф. П. Кесманлы, В. В. Козловский, С. И. Марков, Экспериментальная обработка результатов измерений, СПбГТУ, СПб., 2001. 